\title{
PROBING MACHOs TOWARD THE GALACTIC BULGE
}

\author{
ANDREw Gould \\ Department of Astronomy, Ohio State University, 140 West 18th Avenue, Columbus, OH 43210; gould@astronomy.ohio-state.edu \\ Received 2005 March 27; accepted 2005 May 25
}

\begin{abstract}
If the massive compact halo object (MACHO) fraction of the Galactic dark halo is $f \sim 20 \%$, as suggested by some microlensing experiments, then about $1.2 \%$ of lensing events toward the Galactic bulge are due to MACHOs. For the $40 \%$ of these that lie nearby $\left(D_{l}<4 \mathrm{kpc}\right)$, measurement of their distance $D_{l}$ would distinguish them from bulge lenses, while measurement of their transverse velocity $\boldsymbol{v}_{l}$ would distinguish them from disk lenses. Hence, it would be possible to identify about $0.5 \%(f / 20 \%)$ of all events as due to MACHOs. I show that a planned experiment using the Space Interferometry Mission (SIM PlanetQuest) could thereby detect 1 or 2 such events. This is at the margin of what is required because of a small but nonnegligible background from spheroid stars.
\end{abstract}

Subject headings: dark matter — galaxies: stellar content — gravitational lensing — instrumentation: interferometers

\section{INTRODUCTION}

Following the suggestion of Paczyński (1986), the MACHO (Alcock et al. 1993) and EROS (Aubourg et al. 1993) collaborations began searching for dark matter in the form of massive compact halo objects (MACHOs) by microlensing observations toward the Large Magellanic Cloud (LMC). This target seemed ideal because of the small column of known populations of stars compared to the huge volume of space that would be home to the putative MACHOs. The microlensing optical depth due to known stars was estimated to be $\tau_{\text {disk }}^{\mathrm{LMC}}=8 \times 10^{-9}$ for the Milky Way disk (Gould et al. 1997) and $\tau_{\mathrm{LMC}}^{\mathrm{LMC}}=1 \times 10^{-8}$ for the LMC itself (Gould 1995b). By contrast, if the dark halo were completely composed of MACHOs, their optical depth would be of order

$$
\tau_{\text {full-halo }}^{\mathrm{LMC}} \sim \frac{v_{\mathrm{rot}}^{2}}{c^{2}}=5 \times 10^{-7}
$$

roughly 25 times higher. Here, $v_{\text {rot }}=220 \mathrm{~km} \mathrm{~s}^{-1}$ is the Milky Way rotation speed. Hence, when the experiments began, it seemed as though even a crude measurement of $\tau$ would unambiguously determine whether the halo was composed of MACHOs.

A decade later, the situation is far less clear than was anticipated. MACHO (Alcock et al. 2000) found $\tau \sim 1 \times 10^{-7}$, roughly the geometric mean of the results expected from MACHOs and stars. They interpreted this to mean that the halo was $20 \%$ composed of MACHOs and estimated the typical mass to be $M \sim 0.5 M_{\odot}$. On the other hand, the EROS collaboration (Afonso et al. 2003a; Tisserand \& Milsztajn 2005) found an upper limit for the optical depth due to MACHOs of $5 \%$ of the full-halo value.

One option for resolving this conflict is to explore other lines of sight. Crotts (1992) and Baillon et al. (1993) advocated M31, and several collaborations, including AGAPE (Ansari et al. 1999), Columbia-VATT (Uglesich et al. 2004), MEGA (de Jong et al. 2004), NainiTal (Joshi et al. 2005), POINT-AGAPE (Aurière et al. 2001), SLOTT-AGAPE (Calchi Novati et al. 2003), and WeCAPP (Riffeser et al. 2003), have pursued this suggestion. In many ways this is substantially more challenging than the observations toward the LMC, simply because M31 is 15 times farther away and hence the sources are substantially fainter. Calchi Novati et al. (2005) have just reported a lower limit of $f>10 \%$ with $95 \%$ confidence. This is compatible with the MACHO fraction $f \sim 20 \%$ reported by the MACHO collaboration for the Milky Way halo, but not with the upper limit of $f<5 \%$ reported by EROS.

The microlensing target field that has been monitored the most intensively is the Galactic bulge. Originally proposed by Paczyński (1991) and Griest et al. (1991), major surveys have been carried out by the OGLE (Udalski et al. 1993; Udalski 2003), DUO (Alard et al. 1995), MACHO (Popowski et al. 2005), EROS (Afonso et al. 2003b), and MOA (Abe et al. 2004) collaborations. The primary motivation of both proposals was to probe for disk dark matter and other exotic objects such as a large population of Jupiters. Griest et al. (1991) do mention that if the halo is composed of MACHOs, then these will give rise to an optical depth $\tau_{\text {full-he }}^{\text {bulo }}=1.3 \times 10^{-7}$, but since this is 4 times smaller than the predicted optical depth due to disk stars $\tau_{\text {disk }}^{\text {buge }}=5.1 \times$ $10^{-7}$, there did not appear to be any way to isolate the MACHO events.

Bulge microlensing observations have been enormously fruitful. Kiraga \& Paczyński (1994) showed that the optical depth due to bulge self-lensing was even greater than that due to disk stars. The high event rate encouraged searches for lensing anomalies due to planetary companions of the lenses (Mao \& Paczyński 1991; Gould \& Loeb 1992; Rhie et al. 2000; Albrow et al. 2001b; Gaudi et al. 2002; Abe et al. 2004), which has now yielded the first firm microlensing planet detection (Bond et al. 2004). Bulge microlensing has enabled two microlens mass measurements (An et al. 2002; Kubas et al. 2005) and the probing of bulge-star atmospheres with $\mu$ as resolution both photometrically (Alcock et al. 1997; Albrow et al. 1999, 2000; Fields et al. 2003) and spectroscopically (Castro et al. 2001; Albrow et al. 2001a; Cassan et al. 2004).

Here I show that bulge microlensing can also be used to probe for halo dark matter (MACHOs) in the inner Galaxy. This seems absurd at first sight because the observed optical depth, $\tau_{\text {obs }}^{\text {bulge }} \sim$ $2 \times 10^{-6}$, is about 15 times higher than the rate predicted by Griest et al. (1991), $\tau_{\text {halo }}^{\text {bulge }} \sim 1.3 \times 10^{-7}$, even assuming that the dark halo were completely composed of MACHOs. However, the microlensing experiments toward the LMC and M31 seem to imply that this fraction is perhaps of order $20 \%$, which means that only about $1 \%$ of Galactic bulge microlensing would be due to halo objects. How would one identify these halo microlensing 
events within the barrage of microlensing by ordinary bulge and disk stars?

\section{NEEDLE IN HAYSTACK}

Halo lenses are distinguished from disk lenses by the their transverse velocity $\boldsymbol{v}_{l}$ relative to the Sun, and from bulge lenses by their distance from the Sun, $D_{l}$ (or equivalently, their absolute parallax $\pi_{l}$ ). Hence, to reliably identify the nearby, fast MACHOs, one must reliably measure $\boldsymbol{v}_{l}$ and $\pi_{l}$. Since the MACHOs are by definition "dark" matter, direct observations of the lens cannot be employed in making these determinations, as they were for example for MACHO-LMC-5 (Alcock et al. 2001; Drake et al. 2004; Gould 2004a; Gould et al. 2004). Instead, these quantities must be derived entirely from observations of the source during and after the microlensing events.

\subsection{Observables}

These two quantities $\left(\pi_{l}\right.$ and $\left.\boldsymbol{v}_{l}\right)$ can be expressed in terms of microlensing observables by (e.g., Gould 2000)

$$
\pi_{l}=\pi_{\mathrm{rel}}+\pi_{s}, \quad \pi_{\mathrm{rel}}=\pi_{\mathrm{E}} \theta_{\mathrm{E}}
$$

and

$$
\boldsymbol{v}_{l}=\frac{\boldsymbol{\mu}_{\mathrm{rel}}+\boldsymbol{\mu}_{s}}{\pi_{\mathrm{rel}}+\pi_{s}} \text { AU, } \quad \boldsymbol{\mu}_{\mathrm{rel}}=\frac{\boldsymbol{\theta}_{\mathrm{E}}}{t_{\mathrm{E}}} .
$$

Here, $\pi_{l}, \pi_{s}, \boldsymbol{\mu}_{l}, \boldsymbol{\mu}_{s}$ are the absolute parallaxes and proper motions of the lens and source, $\pi_{\text {rel }}=\pi_{l}-\pi_{s}$ and $\boldsymbol{\mu}_{\text {rel }}=\boldsymbol{\mu}_{l}-\boldsymbol{\mu}_{s}$ are the lens-source relative parallax and proper motion, $\theta_{\mathrm{E}}$ is the angular Einstein radius, $t_{\mathrm{E}}$ is the Einstein timescale, and $\pi_{\mathrm{E}}$ is the microlens parallax (i.e., the inverse of the projected Einstein radius, $\left.\pi_{\mathrm{E}}=\mathrm{AU} / \tilde{r}_{\mathrm{E}}\right)$. The direction of $\boldsymbol{\theta}_{\mathrm{E}}$ is that of the lenssource relative proper motion.

In brief, to determine $\pi_{l}$ and $\boldsymbol{v}_{l}$, one must measure five observables, two 2-vectors $\left(\boldsymbol{\mu}_{s}\right.$ and $\left.\boldsymbol{\theta}_{\mathrm{E}}\right)$ and three scalars $\left(\pi_{s}, \pi_{\mathrm{E}}\right.$, and $\left.t_{\mathrm{E}}\right)$.

\subsection{Parameter Measurement}

Two of the five parameters described in $\S 2.1\left(\pi_{s}\right.$ and $\left.\boldsymbol{\mu}_{s}\right)$ are related solely to the source, while the remaining three $\left(\pi_{\mathrm{E}}, t_{\mathrm{E}}\right.$, and $\left.\boldsymbol{\theta}_{\mathrm{E}}\right)$ are microlensing-event parameters. Of these three, only one $\left(t_{\mathrm{E}}\right)$ is routinely measured during microlensing events. The other two are higher order parameters. While there are a variety of methods to measure $\pi_{\mathrm{E}}$ and $\theta_{\mathrm{E}}$ (see Gould 2001), these generally apply to only a small fraction of events. There are only two events (out of almost 3000 discovered) for which both parameters have been measured from microlensing data alone (An et al. 2002; Kubas et al. 2005), and both of these were binary lenses.

The only known way to routinely determine $\boldsymbol{\theta}_{\mathrm{E}}$ is by highprecision astrometric measurements of the microlensing event (Høg et al. 1995; Miyamoto \& Yoshii 1995; Walker 1995; Paczyński 1998; Boden et al. 1998). The centroid of the microlensed images deviates from the source position by an amount and direction that yields both components of $\boldsymbol{\theta}_{\mathrm{E}}$.

The only known way to routinely determine $\pi_{\mathrm{E}}$ is to make photometric measurements of the event from two locations separated by of order $\tilde{r}_{\mathrm{E}}$ (Refsdal 1966; Gould 1994). The difference in the event parameters then yields both the size of $\tilde{r}_{\mathrm{E}}$ and the direction of motion (the latter potentially confirming the direction extracted from $\left.\boldsymbol{\theta}_{\mathrm{E}}\right)$. Since $\tilde{r}_{\mathrm{E}} \sim O(\mathrm{AU})$, in practice this means placing a satellite in solar orbit. Although there is a fourfold ambiguity in the determination of $\pi_{\mathrm{E}}$, this can be resolved by higher order effects (Gould 1995a). Moreover, measurement of the direction of $\boldsymbol{\theta}_{\mathrm{E}}$ also helps resolve this degeneracy.

\subsection{SIM PlanetQuest Measurements}

Gould \& Salim (1999) showed that the Space Interferometry Mission (SIM PlanetQuest), combined with ground-based photometry, could determine both of these parameters with good $(\sim 3 \%)$ precision with about 5 hours total observation time for bright $(I \sim 15)$ events having typical lens parameters. Moreover, they showed that the same observations would also yield good measurements of $\pi_{s}$ and $\boldsymbol{\mu}_{s}$. Hence, SIM (combined with groundbased photometry) could measure all the required quantities for about 200 events with about 1000 hours of observing time. Indeed, a SIM Key Project has been awarded 1200 hours of observation time to carry out such observations. The main objective of this project is to measure the bulge mass function, but the same observations could cull out the handful of halo events that might be present in the same sample.

As originally designed, SIM was to be a Michelson interferometer with two $35 \mathrm{~cm}$ mirrors (each equivalent to a $D=25 \mathrm{~cm}$ filled aperture) mounted on a $b=10 \mathrm{~m}$ fixed baseline. The width of the interference fringes would then be $\theta \sim \lambda / b=10$ mas, where $\lambda \sim 0.5 \mu \mathrm{m}$ is the central wavelength of the detector. The resulting astrometric precision is $\sim 10 \mathrm{mas} /\left(2 \pi N^{1 / 2}\right)$, where $N$ is the number of photons collected. The design limit of $\sim 4 \mu$ as could therefore be reached in of order a minute for a $V \sim 12$ star. SIM achieves absolute astrometry by monitoring a grid of $\sim 1300$ "grid stars" from which it establishes a global solution with absolute parallax and by tying these to a set of distant quasars to fix the absolute proper motion of this reference frame. All other measurements are made relative to this "grid."

Because SIM astrometry is carried out by counting photons as a function of fringe position (to centroid the fringe), it simultaneously yields total photon counts, i.e., photometry. Both the astrometry and photometry are necessary for microlensing. Because the maximum deviation of the image centroid from the source does not take place until $2^{1 / 2} t_{\mathrm{E}}$ after peak, the astrometric microlensing observations can in principle take place at a fairly leisurely pace. However, the photometric measurements must be initiated quickly in order to accurately measure the time and height of the peak, so that this can be compared precisely to the measurements of these quantities obtained from the ground. The shorter the event, the more rapidly the satellite must respond to the onset of an alert from the ground. At present, the plan is to preschedule observing periods shortly after regular data uploads, during which the spacecraft will be pointed toward the Galactic bulge, but with specific targets "left blank." To accurately monitor short events, these bulge visits must be scheduled frequently, perhaps every 4 or 7 days.

SIM has been descoped since Gould \& Salim (1999) made their analysis. The new performance is not precisely known, but it is likely that the precision will degrade to something like $\sim 5 \%$ for $\pi_{\mathrm{E}}$ and $\sim 10 \%$ for $\theta_{\mathrm{E}}$ for the canonical events considered by Gould \& Salim (1999). Moreover, it is unlikely that $200 I=15$ events will be found during the 5 year primary SIM mission, and using fainter sources (e.g., $I=16.5$ ) would further degrade the precision by a factor 2 . Nevertheless, as I show below, this precision would be quite adequate for distinguishing halo lenses.

The SIM descope could potentially affect the monitoring of halo events in other ways as well. For example, in contrast to the great majority of SIM observations, which take place as the spacecraft slews around the sky in its perpetual monitoring of grid stars, microlensing observations require special slews to and from the bulge for each epoch. If the slew time increases substantially, the penalty for bulge visits can become high, leading to less frequent monitoring and hence reduced sensitivity to short 
events. Thus, how well SIM could actually carry out a search for halo MACHOs toward the bulge depends on a host of technical spacecraft issues that are not yet resolved.

\section{BACKGROUNDS}

\subsection{Spheroid/Bulge Stars}

Halo lenses could produce events anywhere along the line of sight from the Sun to the bulge, and assuming an isothermal halo model with core radius $a=5 \mathrm{kpc}$, the density

$$
\rho_{\text {halo }}=\frac{v_{c}^{2}}{4 \pi G\left(R^{2}+a^{2}\right)}
$$

rises all the way to the Galactic center. Here $R$ is Galactocentric distance. The optical depth per unit path length along a line of sight toward the Galactic center therefore also rises almost all the way in

$$
\frac{d \tau_{\mathrm{MACHOs}}^{\text {bulge }}}{d D_{l}}=\frac{f \rho_{\text {halo }}}{M} \pi r_{\mathrm{E}}^{2}=\frac{v_{c}^{2}}{c^{2}} \frac{f}{R_{0}} \frac{x(1-x)}{\left(a / R_{0}\right)^{2}+(1-x)^{2}} .
$$

Here, $r_{\mathrm{E}}=\left(4 G M D_{l} D_{l s} / c^{2} D_{s}\right)^{1 / 2}$ is the Einstein radius, $D_{l}$ and $D_{s}$ are the source and lens distances, $D_{l s}=D_{s}-D_{l}, f$ is the fraction of the halo in the form of MACHOs, $R_{0}=8 \mathrm{kpc}$ is the solar Galactocentric distance, $x \equiv D_{l} / R_{0}$, and $M$ (which cancels out) is the mass of the lens. ${ }^{1}$

However, in the inner Galaxy, these halo lenses are completely submerged in the background of bulge lenses, and since they have similar kinematics, there is no way to reliably distinguish them. It is only out closer to the Sun, where the spheroidal population (here usually called "spheroid" or "stellar halo") thins out, that one may hope to separate the two populations. Even here, there is some possibility of contamination. The local spheroid density is only about $1 \%$ of the dark halo, but if $f \sim 20 \%$, as Alcock et al. (2000) suggest, then only 20 times more mass is locked up in MACHOs than in local spheroid stars. Moreover, as one approaches the Galactic center, the spheroid density grows substantially more rapidly than does the dark halo. To make a quantitative comparison, I adopt

$$
\rho_{\text {spheroid }}=1 \times 10^{-4} \frac{M_{\odot}}{\mathrm{pc}^{3}}\left(\frac{R}{R_{0}}\right)^{-\nu}, \quad \nu=3.2 .
$$

After accounting for observed stars and extrapolating down to brown dwarfs and up to the progenitors of remnants, Gould et al. (1998) estimate $6.4 \times 10^{-5} M_{\odot} \mathrm{pc}^{-3}$ for the density of the spheroid in the solar neighborhood. However, both Dahn et al. (1995) and Gould (2003) find substantially more low-luminosity $\left(M_{V}>8\right)$ stars than did Gould et al. (1998) in their more local samples (see Fig. 2 from Gould 2004b), so I have adjusted the Gould et al. (1998) estimate upward. The power-law slope is measured by several techniques (Gould et al. 1998 and references therein).

Figure 1 shows the optical depth per unit distance due to spheroid stars and to putative MACHOs under the assumption

\footnotetext{
${ }^{1}$ Note that I have made the simplification that $D_{s}=R_{0}$. This is appropriate because the lenses in question lie well in the foreground, which means that there is no "bias" toward higher source distance, so that the sources will be sharply-and symmetrically - peaked around the Galactocentric distance. In fact, because the bar is inclined to the line of sight, the distance to the source-density peak will vary monotonically with Galactic longitude. However, it remains true that for foreground lenses, the mean source distance (averaged over many lines of sight) will be $R_{0}$, whereas it is higher than $R_{0}$ for bulge lenses.
}

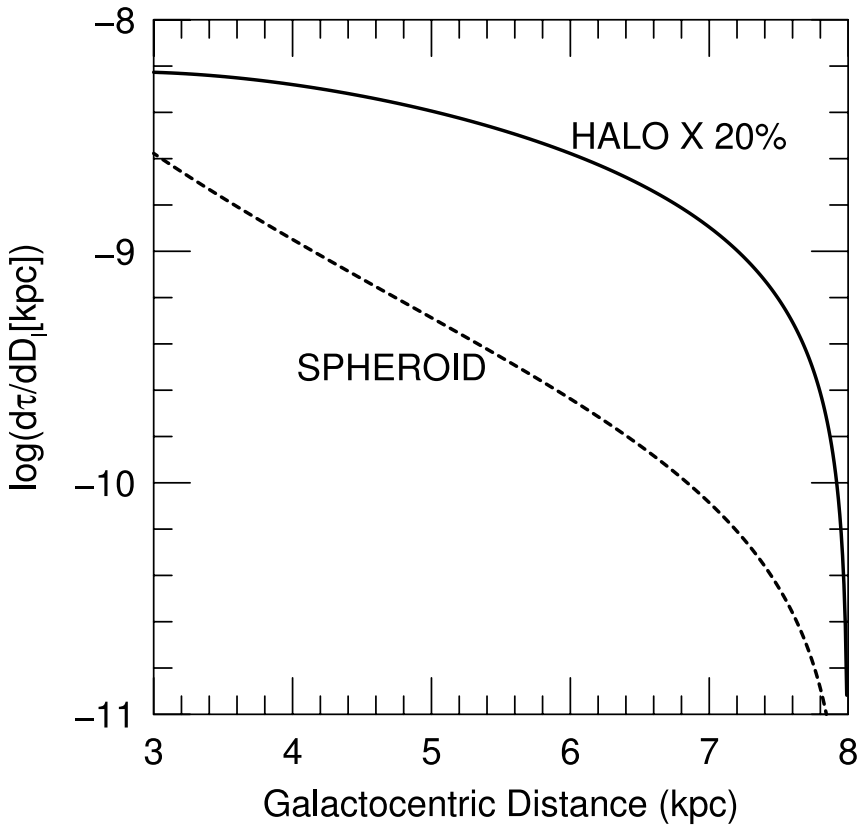

FIG. 1.-Optical depth per unit path length $d \tau / d D_{l}$ as a function of distance from the Galactic center for a source near the Galactic center. The halo (assuming a $f=20 \%$ MACHO fraction) and the spheroid are shown by solid and dashed curves, respectively. For $f=20 \%$, spheroid stars are a $20 \%$ background at $R=4 \mathrm{kpc}$ and a $10 \%$ background at $R=7 \mathrm{kpc}$, which implies that 2 or 3 halo lenses must be identified at $R>4 \mathrm{kpc}$ for a reliable halo "detection." Inside $R<4 \mathrm{kpc}$ the spheroid continues to grow (and also transforms into the bulge), making the identification of halo lenses less secure. Hence, the experiment should be restricted to $R>4 \mathrm{kpc}$, where the total optical depth is $\tau=5 \times 10^{-8} f$.

that $f=20 \%$. It shows that even with a MACHO fraction of $20 \%$, the halo dominates the spheroid from $R=R_{0}$ to $R=4 \mathrm{kpc}$, which latter is about the limit to which the local spheroid density profile can be reliably extrapolated. However, this domination is not overwhelming: at $R=4 \mathrm{kpc}$ it is only a factor of 5, and even at $R=7 \mathrm{kpc}$ (where the halo optical depth has fallen by a factor 5) the halo only dominates by a factor 10 . This means that 2 or 3 halo lenses would have to be identified to constitute a reliable "MACHO detection." Otherwise, there would be a significant possibility that spheroid lenses were responsible.

Since one must restrict attention to $D_{l}<4 \mathrm{kpc}$, the total available halo optical depth is reduced by a factor 0.4 relative to the $1.3 \times 10^{-7}$ calculated by Griest et al. (1991). If we further assume $f=20 \%$, the available halo optical depth is further reduced to $10^{-8}$, about $0.5 \%$ of the observed optical depth toward the Galactic bulge of $\tau \sim 2 \times 10^{-6}$ (Afonso et al. 2003b; Popowski et al. 2005; Sumi et al. 2005). Hence, assuming for the moment that the event rates are in proportion to the optical depths, roughly 200 measurements would be required to identify a single halo lens. Thus, if the SIM mission were extended from 5 to 10 years (as is currently envisioned), then one might expect to find about 2 halo lenses. As noted above, this is just at the margin of a viable detection.

If a handful of putative MACHO events were found, a possible alternative explanation would be that the contribution of the spheroid had been underestimated. For example, the spheroid might have a steeper power law than I have assumed, but even at $\nu=3.5$ the spheroid density at $4 \mathrm{kpc}$ would only rise by a factor 1.25 . The spheroid could in principle have a much higher normalization than given by equation (6), but unless the several groups responsible for counting spheroid stars have all been grossly incompetent, such a higher normalization could only arise 
from a heretofore unrecognized dark spheroid component. It would be virtually impossible to distinguish such a component from halo MACHOs based on objects detected near the Sun and, in any case, would be a major discovery.

\subsection{Thick-Disk Stars}

Since halo objects travel in random orbits, some will, by chance, have transverse velocities that are indistinguishable from those of disk stars. However, since the phase-space distribution of disk stars is quite confined, the fraction of halo objects that could be confused with disk stars is extremely small and can be ignored. The same does not hold for the thick disk, which, while of much lower density than the thin disk, spans a much larger region of velocity space. The division of the "disk" into "thin" and "thick" components is somewhat arbitrary. In the present context, the greatest concern is regarding the hottest component of the thick disk, so I will adopt the 4\% thick-disk/disk normalization (at the Sun) of Kuijken \& Gilmore (1989), whose thick disk is fairly hot. Adopting a local thin-disk normalization of $\rho_{0 \text {, disk }} \sim 0.037 M_{\odot} \mathrm{pc}^{-3}$ (Zheng et al. 2001 and references therein), this implies that the thick disk is locally about 15 times denser than the spheroid. Because the lensing signal is dominated by MACHOs lying $\sim 4 \mathrm{kpc}$ toward the Galactic center (see Fig. 1), it is more appropriate to make the comparison at that distance, where the thick disk is thicker by a factor $\exp (4 \mathrm{kpc} / 2.7 \mathrm{kpc}) \sim 4$ but the spheroid is thicker by a factor $(8 \mathrm{kpc} / 4 \mathrm{kpc})^{3.2} \sim 9$. This still implies that the thick disk is about 7 times denser than the spheroid and so a factor $\sim 1.5$ denser than an $f \sim 20 \%$ MACHO halo. Hence, this background cannot be ignored and must be eliminated by kinematic cuts.

Qualitatively speaking, to be in any type of "disk" thick-disk stars must be in prograde orbits, whereas half of MACHOs would be on retrograde orbits. Hence, half of MACHO phase space is clearly free of thick-disk contamination. To estimate more precisely the amount of phase space that would be "effectively free" of contamination, I model the thick disk (viewed toward the Galactic bulge) by Gaussian dispersions of $60 \mathrm{~km} \mathrm{~s}^{-1}$ and $40 \mathrm{~km} \mathrm{~s}^{-1}$ in the horizontal and vertical directions and by an asymmetric drift of $30 \mathrm{~km} \mathrm{~s}^{-1}$. Hence, $95 \%$ of thick disk stars have transverse velocities lying in an ellipse with semiaxes of $150 \mathrm{~km} \mathrm{~s}^{-1}$ and $100 \mathrm{~km} \mathrm{~s}^{-1}$ centered on $(190,0) \mathrm{km} \mathrm{s}^{-1}$ in the Galactic frame. I find that $<15 \%$ of MACHOs lie in this ellipse, independent of whether I choose halo dispersions of $v_{\text {rot }} / 2^{1 / 2}$ or $v_{\text {rot }} / 3^{1 / 2}$. Hence, contamination by the thick disk poses a relatively minor problem.

\subsection{Vetting Spheroid Interlopers with High-Resolution Imaging}

As described in $\S 3.1$, there is a small but nonnegligible chance that an ordinary spheroid star could masquerade as a MACHO. However, from the event solution, one would know the lens mass and distance, which would allow one to predict its flux (on the assumption that it was a subdwarf). This flux would enter $F_{b}$, the blended flux, which is one of the parameters of a microlensing fit. If the predicted lens flux were significantly greater than the measured $F_{b}$, this would rule out the spheroid-subdwarf hypothesis. ${ }^{2}$ Of course, it is also possible that the implied lens flux will be consistent with the measured $F_{b}$. Moreover, even if it

\footnotetext{
2 SIM's $10 \mathrm{~m}$ baseline will make it sensitive to point sources in the region around the source with a resolution of 10 mas, substantially better than the Hubble Space Telescope. Bennett et al. (2002) used HST to resolve the blended light for microlensing black hole candidates, and thereby substantially constrained the possibility that the lenses were luminous.
}

were not, it would still be possible that the lens was a spheroid white dwarf.

However, in these cases, one can still make an additional test using high-resolution imaging (employing either adaptive optics or a space-based imager). MACHO events (and the spheroid events that simulate them) will typically have very high lenssource relative proper motions, $\mu_{\text {rel }} \sim 300 \mathrm{~km} \mathrm{~s}^{-1} / 4 \mathrm{kpc} \sim$

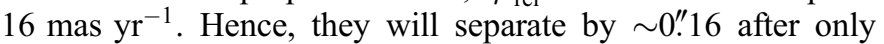
10 years. Moreover, since the direction and magnitude of this proper motion will be known, the position of the lens relative to the source will be known quite accurately at any future epoch. Hence, for any given instrumental setup, one will be able to specify the limiting magnitude to which the source could be detected. Although the sources will necessarily be bright, $I \lesssim 16.5$ (otherwise SIM could not monitor them at all), by waiting sufficiently long, one could test not only for spheroid stars at the subdwarf luminosity corresponding to the measured mass, but also for much dimmer objects such as old white dwarfs. With the ability to test for such faint backgrounds, one could enhance the statistical significance of the detection of MACHO candidates, even if there are only 1 or 2 of them.

\section{PRACTICAL CONSIDERATIONS}

\subsection{Event Timescales}

The Einstein timescales of these halo events are given by

$$
t_{\mathrm{E}}=16 \text { days }\left[\frac{M}{0.5 M_{\odot}} \frac{x(1-x)}{0.25}\right]^{1 / 2}\left(\frac{v_{\perp}}{300 \mathrm{~km} \mathrm{~s}^{-1}}\right)^{-1}
$$

where $v_{\perp}$ is the transverse lens velocity relative to the observersource line of sight. Thus, for the mass range advocated by Alcock et al. (2000), the typical event timescales will be fairly short. This is important because the event must be identified and alerted to the satellite well before peak in order to measure $\pi_{\mathrm{E}}$ (Gould \& Salim 1999). Hence, a fairly aggressive posture is required to keep the halo events in the sample.

However, the fact that these halo events are somewhat shorter than typical bulge events means that they are also more frequent than would be indicated by their optical depth alone. That is, the event rate $\Gamma \propto \tau / t_{\mathrm{E}}$, so the rate is inversely proportional to the timescale. Hence, the shorter timescales enhance the viability of a given experiment relative to what was discussed in $\S 3.1$, provided that not too many halo events are lost because they are too short.

\subsection{Signal-to-Noise Ratios}

The two microlensing parameters being measured are related to the underlying physical parameters by

$$
\pi_{\mathrm{E}}=\sqrt{\frac{\pi_{\mathrm{rel}}}{\kappa M}}, \quad \theta_{\mathrm{E}}=\sqrt{\kappa M \pi_{\mathrm{rel}}}
$$

where $\kappa=4 G / \mathrm{AU} c^{2} \sim 8.1$ mas $M_{\odot}^{-1}$. Hence, for fixed $M$, both $\pi_{\mathrm{E}}$ and $\theta_{\mathrm{E}}$ are proportional to $\pi_{\mathrm{rel}}^{1 / 2}$. Since the absolute errors in these two quantities are approximately independent of their size, this means that the fractional errors decline as $\pi_{\text {rel }}^{-1 / 2}$. The basic experiment is designed for typical bulge-bulge lensing, in which the lenses are of order $M \sim 0.5 M_{\odot}$ and the relative parallaxes are $\pi_{\text {rel }} \sim \mathrm{AU} / 7 \mathrm{kpc}-\mathrm{AU} / 9 \mathrm{kpc}=31 \mu \mathrm{as}$. By contrast, since $D_{l} \leq 4 \mathrm{kpc}$, the halo-lens relative parallaxes are $\pi_{\text {rel }}>125 \mu \mathrm{as}=$ $\mathrm{AU} / 4 \mathrm{kpc}-\mathrm{AU} / 8 \mathrm{kpc}$. Hence, if a halo event is successfully 
monitored, both $\pi_{\mathrm{E}}$ and $\theta_{\mathrm{E}}$ will be measured substantially more accurately than for typical events. ${ }^{3}$

\section{DISCUSSION}

In this paper, I have considered microlensing by a "standard" spherical isothermal halo and have demonstrated that it is marginally possible to detect such a halo in an already-planned experiment using SIM, even in the face of backgrounds from the Galactic spheroid and thick disk.

As first pointed out by Sackett \& Gould (1993), however, anisotropic halos (that remain normalized to reproduce the rotation curve) can dramatically alter the microlensing optical depth toward various lines of sight. For example, Sackett \& Gould (1993) argued that a flattened halo would enhance the optical depth toward the LMC relative to the SMC, because the latter is at higher Galactic latitude. More precisely, they found that the

\footnotetext{
3 Again note that in bulge-bulge lensing, there is a "bias" that pushes the sources behind the peak of the bulge density profile, whereas for lensing by foreground objects, any such bias is extremely weak. For this reason "typical" bulge-bulge sources are placed at $9 \mathrm{kpc}$ whereas "typical" MACHO-event sources are placed at $8 \mathrm{kpc}$.
}

optical depth toward the LMC was approximately independent of the flattening, while the SMC value fell as the flattening increased. The same trend continues for the bulge, which of course lies at extremely low latitudes. For locations close to the Galactic plane, the halo density is enhanced relative to the spherical case by a factor (Sackett \& Gould 1993, eq. [2.1])

$$
\frac{\rho_{\text {halo }}}{\rho_{\text {sph-halo }}}=\frac{\tan \psi}{\psi}, \quad \cos \psi=q \text {, }
$$

where $q$ is the axis ratio of the halo mass distribution. Hence, for $q=0.4$ this enhancement is roughly a factor 2 . Given the fact that any halo detection made in the first-generation experiment discussed here will be dominated by Poisson statistics, it will probably not be possible to determine the halo flattening parameter this way, but this may become possible in future experiments.

The paper was greatly improved by the comments and suggestions of the referee, Piotr Popowski. This work was supported by grant AST 02-01266 from the NSF and by JPL contract 1226901 .
Abe, F., et al. 2004, Science, 305, 1264

Afonso, C., et al. 2003a, A\&A, 400, 951 2003b, A\&A, 404, 145

Alard, C., Mao, S., \& Guibert, J. 1995, A\&A, 300, L17

Albrow, M. D., et al. 1999, ApJ, 512, 672

2000, ApJ, 534, 894

2001a, ApJ, 550, L173

2001b, ApJ, 556, L113

Alcock, C., et al. 1993, Nature, 365, 621

1997, ApJ, 491, 436

2000, ApJ, 542, 281

2001, Nature, 414, 617

An, J. H., et al. 2002, ApJ, 572, 521

Ansari, R., et al. 1999, A\&A, 344, L49

Aubourg, E., et al. 1993, Nature, 365, 623

Aurière, M., et al. 2001, ApJ, 553, L137

Baillon, P., Bouquet, A., Giraud-Héraud, Y., \& Kaplan, J. 1993, A\&A, 277, 1

Bennett, D. P., et al. 2002, ApJ, 579, 639

Boden, A. F., Shao, M., \& Van Buren, D. 1998, ApJ, 502, 538

Bond, I. A., et al. 2004, ApJ, 606, L155

Calchi Novati, S., et al. 2003, A\&A, 405, 851 2005, A\&A, submitted (astro-ph/0504188)

Cassan, A., et al. 2004, A\&A, 419, L1

Castro, S., Pogge, R. W., Rich, R. M., DePoy, D. L., \& Gould, A. 2001, ApJ, 548, L197

Crotts, A. P. S. 1992, ApJ, 399, L43

Dahn, C. C., Liebert, J. W., Harris, H., \& Guetter, H. C. 1995, The Bottom of the

Main Sequence and Beyond, ed. C. G. Tinney (Heidelberg: Springer), 239

Drake, A. J., Cook, K. H., \& Keller, S. C. 2004, ApJ, 607, L29

de Jong, J. T. A., et al. 2004, A\&A, 417, 461

Fields, D. L., et al. 2003, ApJ, 596, 1305

Gaudi, B. S., et al. 2002, ApJ, 566, 463

Gould, A. 1994, ApJ, 421, L75

1995a, ApJ, 441, L21

1995b, ApJ, 441, 77
REFERENCES

Gould, A. 2000, ApJ, 542, 785

2001, PASP, 113, 903

2003, ApJ, 583, 765

2004a, ApJ, 606, 319

2004b, ApJ, 607, 653

Gould, A., Bahcall, J. N., \& Flynn, C. 1997, ApJ, 482, 913

Gould, A., Bennett, D. P., \& Alves, D. R. 2004, ApJ, 614, 404

Gould, A., Flynn, C., \& Bahcall, J. N. 1998, ApJ, 503, 798

Gould, A., \& Loeb, A. 1992, ApJ, 396, 104

Gould, A., \& Salim, S. 1999, ApJ, 524, 794

Griest, K., et al. 1991, ApJ, 372, L79

Høg, E., Novikov, I. D., \& Polanarev, A. G. 1995, A\&A, 294, 287

Joshi, Y. C., Pandey, A. K., Narasimha, D., \& Sagar, R. 2005, A\&A, 433, 787

Kiraga, M., \& Paczyński, B. 1994, ApJ, 430, L101

Kubas, D., et al. 2005, A\&A, 435, 941

Kuijken, K., \& Gilmore, G. 1989, MNRAS, 239, 605

Mao, S., \& Paczyński, B. 1991, ApJ, 374, L37

Miyamoto, M., \& Yoshii, Y. 1995, AJ, 110, 1427

Paczyński, B. 1986, ApJ, 304, 1 1991, ApJ, 371, L63 1998, ApJ, 494, L23

Popowski, P., et al. 2005, ApJ, 631, in press

Refsdal, S. 1966, MNRAS, 134, 315

Rhie, S. H., et al. 2000, ApJ, 533, 378

Riffeser, A., Fliri, J., Bender, R., Seitz, S., \& Gössl, C. A. 2003, ApJ, 599, L17

Sackett, P. D., \& Gould, A. 1993, ApJ, 419, 648

Sumi, T., et al. 2005, ApJ, submitted (astro-ph/0502363)

Tisserand, P., \& Milsztajn, A. 2005, Proc. 5th Rencontres du Vietnam, New Views on the Universe, in press (astro-ph/0501584)

Udalski, A. 2003, Acta Astron., 53, 291

Udalski, A., Szymański, M., Kałużny, J., Kubiak, M., Krzemiński, W., Mateo, M., Preston, G. W., \& Paczyński, B. 1993, Acta Astron., 43, 289

Uglesich, R. R., et al. 2004, ApJ, 612, 877

Walker, M. A. 1995, ApJ, 453, 37

Zheng, Z., Flynn, C., Gould, A., Bahcall, J. N., \& Salim, S. 2001, ApJ, 555, 393 